\title{
Macrodrenagem urbana: comparativo de hidrogramas gerados pelo método precipitação vazão, Soil Conservation Service (SCS), entre duas equações de chuva de boa confiabilidade
}

\section{Urban macrodrainage: comparative of hydrograms generated by the rainfall-runoff method, Soil Conservation Service among two good reliability rainfall equations}

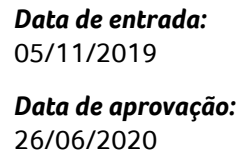

André Carvalho Coelho $^{1 *}$ | Antonio Carlos Zuffo ${ }^{1}$

DOI: https://doi.org/10.36659/dae.2021.076

ORCID ID

Coelho AC (ID) https://orcid.org/0000-0003-0172-680X

Zuffo AC (iD) https://orcid.org/0000-0002-2186-9755

\section{Resumo}

Este trabalho utiliza duas equações de chuva para calcular a vazão de pico de uma bacia hidrográfica localizada na região leste da cidade de São Paulo, a bacia do córrego Tiquatira. O modelo computacional utilizado, o HEC-HMS, permite realizar as simulações hidrológicas utilizando um método de precipitação vazão muito utilizado, o Soil Conservation Service (SCS). Na região da bacia em estudo, ocorrem frequentes inundações devido ao transbordamento do córrego Tiquatira, então se destaca a importância de utilizar a equação de chuva mais apropriada para a região, a fim de que um novo projeto de drenagem não subestime as estruturas hidráulicas a serem construídas. A equação de chuva desenvolvida por Martinez e Magni (1999) é bastante utilizada para os projetos de drenagem na cidade de São Paulo, portanto é uma equação de boa confiabilidade e consolidada. Porém a equação de chuva desenvolvida por Zuffo (2009), para a cidade de Guarulhos, apresenta maiores taxas de intensidades pluviométricas; ressalta-se que o posto pluviográfico de origem dos dados para o desenvolvimento dessa equação encontra-se próximo à região de estudo. Portanto foi apresentado o impacto que pode representar na vazão de pico utilizando as equações que inicialmente seriam recomendadas em uma bacia hidrográfica da região leste da cidade de São Paulo.

Palavras-chave: Modelos hidrológicos. Urbanização. HEC-HMS. Equações de chuva.

\section{Abstract}

This work compares the use of two rainfall equations to calculate the peak flow of a watershed located in the eastern region of the city of São Paulo, the Tiquatira stream basin. The computational model used, the HEC-HMS, allows to perform the hydrological simulations using a widely used rainfall-runoff method, the Soil Conservation Service (SCS). In the basin region under study, frequent flooding occurs due to the overflow of the Tiquatira stream, so the importance of using the most appropriate rainfall equation for the region is highlighted, so that

\footnotetext{
1 Unicamp (Universidade Estadual de Campinas) - Campinas - São Paulo - Brasil.

* Autor correspondente: andrecoelho.engenhariaagmail.com.
} 
a new drainage project does not underestimate the hydraulic structures to be be built. The rainfall equation developed by Martinez and Magni (1999) is widely used for drainage projects in the city of São Paulo, therefore, it is a good reliable and consolidated equation. However, the rainfall equation developed by Zuffo (2009) for the city of Guarulhos, presents higher rates of rainfall intensities, and it is noteworthy that the rainfall source of data for the development of the equation is close to the study region. Therefore, the impact that can represent on the peak flow was presented using the equations that would initially be recommended in a watershed of the eastern region of the city of São Paulo.

Keywords: Hydrological models. Urbanization. HEC-HMS. Rainfall equations.

\section{INTRODUÇÃO}

Na cidade de São Paulo, durante o século XX, houve um grande aumento da urbanização, que, sem planejamento, levou a uma alta taxa de impermeabilização, alterando o microclima da cidade e fazendo com que as águas das chuvas que infiltravam no solo escoassem superficialmente e causassem as frequentes inundações, principalmente durante o verão. Neste período ocorrem as chuvas convectivas, popularmente conhecidas como chuvas de verão, formadas por meio de um processo de convecção de massa de ar úmido, na qual a radiação solar aquece o solo e a massa de ar. Durante o período da tarde, quando ocorre a diminuição da radiação solar, pelo contato da radiação da massa de ar com o solo e por conta do calor armazenado, é criada uma situação de instabilidade e, então, formamse as chuvas de alta intensidade e curta duração (Tucci et al,. 2015). Esses eventos de chuva de alta intensidade ocasionam as inundações por conta da impermeabilização da bacia, com destaque para os prejuízos materiais, doenças e até perdas de vidas sofridas principalmente pela parcela da população de baixa renda, sujeita a políticas insuficientes de habitação e sem recursos financeiros que possibilitem adquirir os terrenos das áreas formais urbanizadas das cidades, os quais são de alto valor. Sem opções, resta a parte dessa população habitar os espaços vazios das cidades, geralmente associados às fragilidades ambientais como as áreas de bai- xada, próximas às margens dos rios e córregos, conforme Nunes, Silva e Fonseca (2017).

O fato citado torna os projetos de drenagem urbana de grande relevância, levando os especialistas a utilizar, de forma criteriosa, os métodos contemplados na literatura dos estudos hidrológicos conforme as características da região em estudo. Uma das etapas de fundamental importância é a escolha da equação de chuva conforme as características do local, provenientes de dados pluviográficos ou pluviométricos.

Para a cidade de São Paulo, 6 equações estão publicadas no estudo realizado pelo Departamento de Águas e Energia Elétrica (DAEE) em conjunto com o Centro Tecnológico de Hidráulica (CTH), "Precipitações intensas na bacia do Alto Tietê", publicado por São Paulo (2018), na qual a Equação de Martinez e Magni (1999) é bastante utilizada para projetos de drenagem da região, sendo uma equação bem consolidada. Porém o presente estudo ressalta a importância da verificação, neste caso, para uma bacia localizada na região leste da cidade de São Paulo, usando a Equação de Zuffo (2009) para Guarulhos, também publicada por São Paulo (2018). Essa última equação utilizou 21 anos de dados da Estação Pluviográfica de Cumbica e a mais próxima à bacia do córrego Tiquatira em estudo em comparação com o posto IAG-USP. Na disponibilidade de mais de uma equação de chuva para uma determinada região, é recomendado utilizar os maiores valo- 
res de intensidades de precipitação, de acordo com São Paulo (2018).

Objeto de estudo, a bacia do córrego Tiquatira está localizada na região leste da cidade de São Paulo, nas coordenadas 7.394 .678 a $7400426 \mathrm{~N}$ e de 341.182 a 349.638 E (UTM, Zona 23), e nela estão importantes bairros pela sua população, como a Penha, São Miguel e Itaquera, além da avenida Governador Carvalho Pinto, localizada às margens do córrego Tiquatira. Para a realização do estudo, as seguintes etapas foram obedecidas. 0 mapa ilustrado pela Fig. 1 indica a localização das estações utilizadas no estudo em relação à bacia do córrego Tiquatira, sendo as distâncias de 9,6 km para o posto da INFRAERO e $19,7 \mathrm{~km}$ para o posto IAG-USP.

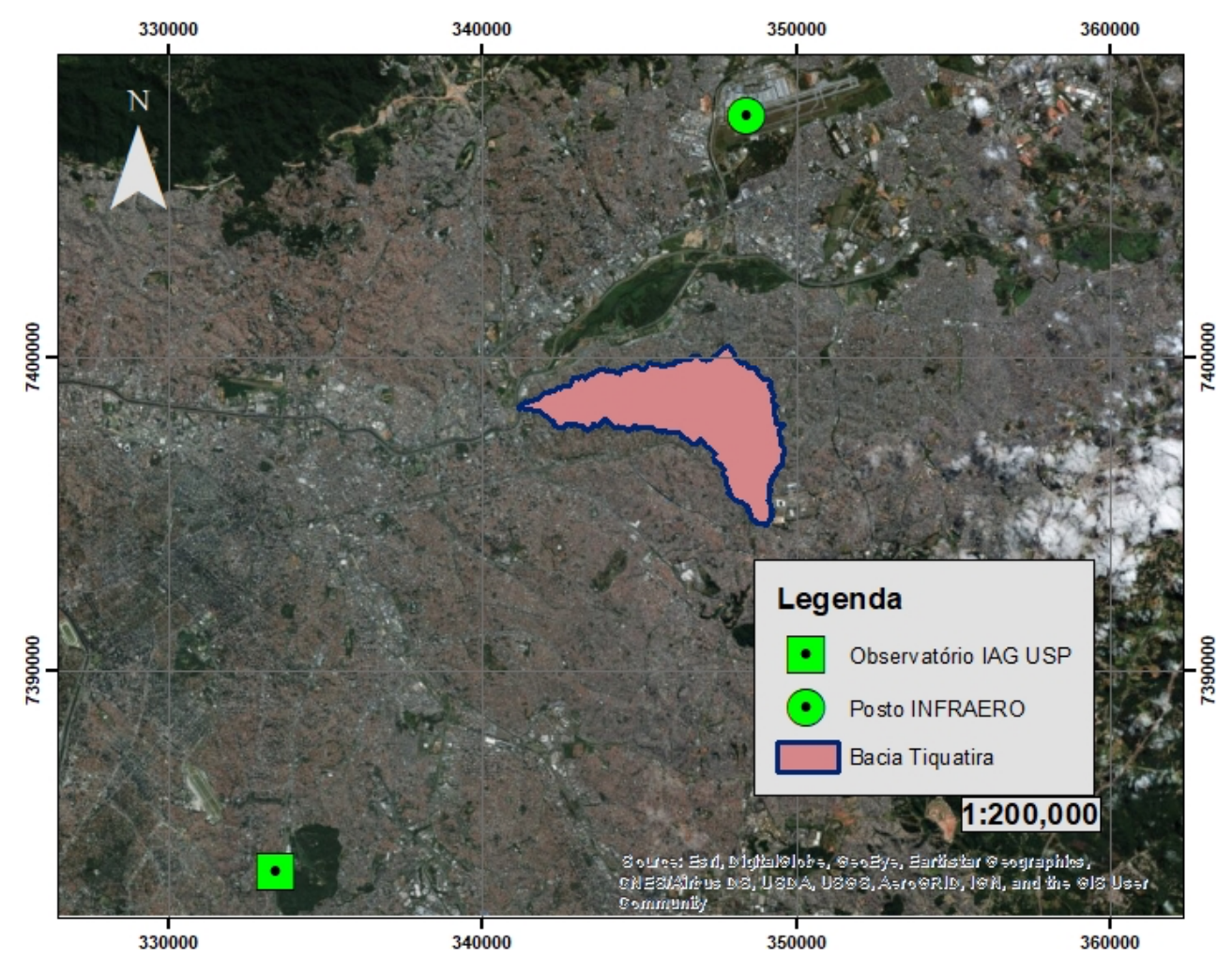

Figura 1 - Mapa dos postos pluviográficos em relação à bacia do córrego Tiquatira.

A Equação de Martinez e Magni (2015), conforme São Paulo (2018), utilizou dados de 1933-36, $1938,1940-45,1948-61,1963-73,1975,1977-$ 82, 1984-98, 2001-05, 2008, 2009 e 2011, totalizando 65 anos. A equação relaciona intensidade, duração e frequência das precipitações e incorpora a equação proposta por Ven-Te-Chow (1954), conforme a Eq. 1:

$$
i_{t, T}=M_{(i) t}+\sigma_{(i) t} k_{n, T}
$$

Após realizada a leitura dos pluviógrafos pelo "Método do Ponto de Inflexão", conforme São
Paulo (2018), que proporciona a rápida leitura e codificação, incluindo boa precisão de 10 em 10 minutos, os dados foram analisados pelo $\mathrm{CTH}$ para a correção de possíveis erros e assim geradas as séries históricas para máximas intensidades de chuvas padronizadas para durações de 10 até 1440 minutos. Posteriormente foram definidos os anos a serem contemplados, em seguida a verificação do ajuste a distribuição de Gumbel, e então a Equação de Martinez e Magni (1999) foi definida conforme a Eq. 2: 
$i_{t, T}=39,30(t+20)^{-0,9228}+10,18(t+20)^{-0,8764}\left[-0,4653-0,8407 \ln \ln \left[\frac{T}{T-1}\right]\right.$

Para $10 \leq \mathrm{t} \leq 1440 \mathrm{~min}$.

Em que:

i: intensidade da chuva em $\mathrm{mm} /$ minutos

t: duração da chuva em minutos

$\mathrm{T}$ : período de retorno em anos

A Tabela 1 indica os resultados da previsão de máximas intensidades de chuvas para a cidade de São Paulo:

Tabela 1 - Previsão de máximas intensidades de chuvas, em mm/h, pelo método de Ven-Te-Chow (1954).

\begin{tabular}{|c|c|c|c|c|c|c|c|}
\hline \multirow{2}{*}{$\begin{array}{c}\text { Duração } \\
\text { (minutos) }\end{array}$} & \multicolumn{7}{|c|}{ TR (anos) } \\
\hline & 5 & 10 & 15 & 20 & 25 & 50 & 100 \\
\hline 10 & 126,87 & 146,42 & 157,46 & 165,18 & 171,13 & 189,47 & 207,66 \\
\hline 15 & 110,20 & 127,28 & 136,92 & 143,67 & 148,87 & 164,89 & 180,78 \\
\hline 20 & 97,54 & 112,74 & 121,31 & 127,32 & 131,94 & 146,19 & 160,33 \\
\hline 30 & 79,55 & 92,05 & 99,10 & 104,04 & 107,84 & 119,56 & 131,19 \\
\hline 45 & 62,60 & 72,53 & 78,13 & 82,05 & 85,08 & 94,39 & 103,63 \\
\hline 60 & 51,78 & 60,06 & 64,73 & 68,00 & 70,52 & 78,28 & 85,98 \\
\hline 120 & 31,06 & 36,13 & 38,99 & 40,99 & 42,54 & 47,29 & 52,00 \\
\hline 180 & 22,43 & 26,13 & 28,23 & 29,69 & 30,82 & 34,30 & 37,75 \\
\hline 240 & 17,65 & 20,60 & 22,26 & 23,42 & 24,32 & 27,08 & 29,82 \\
\hline 300 & 14,60 & 17,06 & 18,44 & 19,41 & 20,16 & 22,46 & 24,75 \\
\hline 360 & 12,48 & 14,59 & 15,79 & 16,62 & 17,26 & 19,24 & 21,21 \\
\hline 420 & 10,92 & 12,78 & 13,82 & 14,56 & 15,12 & 16,87 & 18,59 \\
\hline 480 & 9,72 & 11,38 & 12,31 & 12,97 & 13,48 & 15,03 & 16,58 \\
\hline 600 & 7,98 & 9,36 & 10,14 & 10,68 & 11,10 & 12,39 & 13,67 \\
\hline 720 & 6,79 & 7,97 & 8,64 & 9,10 & 9,46 & 10,56 & 11,66 \\
\hline 960 & 5,26 & 6,18 & 6,70 & 7,06 & 7,34 & 8,21 & 9,06 \\
\hline 1200 & 4,30 & 5,06 & 5,49 & 5,79 & 6,03 & 6,74 & 7,45 \\
\hline 1440 & 3,65 & 4,30 & 4,67 & 4,93 & 5,12 & 5,73 & 6,34 \\
\hline
\end{tabular}

Fonte: São Paulo (2018) adaptada

Os dados pluviográficos para a Equação de Guarulhos são provenientes da estação meteorológica mantida pela INFRAERO desde 1987, com leituras realizadas automaticamente em intervalos de 1 em 1 hora Zuffo (2009). O método proposto por Chen (1983) foi o que melhor representou os dados, portanto foi utilizado para determinar as intensidades para obtenção da Eq. 3:

$$
R_{t}^{T}=\frac{a_{1} * R_{1}^{10} * \log \left(10^{2-x} * T^{x-1}\right.}{\left(t+b_{1}\right)^{c_{1}}} *\left(\frac{t}{60}\right)
$$

Zuffo (2009) desenvolveu 4 equações de chuvas para a cidade de Guarulhos, sendo apresentada na Eq. 4 aquela cuja fórmula é a mais simplificada,

$$
i=\frac{2507 * T_{R}^{0,1748}}{(T+19)^{0,8958 * T_{R}^{0,0053}}}
$$

$\mathrm{Na}$ qual os intervalos de validade estão compreendidos para os valores:

5 minutos $\leq \mathrm{t} \leq 1440$ minutos ( $1 \mathrm{dia}$ );

5 anos $\leq T R \leq 100$ anos.

A Tabela 2 indica os resultados da previsão de máximas intensidades de chuvas para a cidade de Guarulhos. 
Tabela 2 - Previsão de máximas intensidades de chuvas, em mm/h, pelo método de Chen (1983)

\begin{tabular}{|c|c|c|c|c|c|c|c|}
\hline \multirow{2}{*}{$\begin{array}{c}\text { Duração } \\
\text { (minutos) }\end{array}$} & \multicolumn{7}{|c|}{ TR (anos) } \\
\hline & 5 & 10 & 15 & 20 & 25 & 50 & 100 \\
\hline 10 & 158,52 & 176,95 & 188,70 & 197,51 & 204,62 & 228,39 & 254,90 \\
\hline 15 & 137,30 & 153,18 & 163,31 & 170,89 & 177,01 & 197,47 & 220,27 \\
\hline 20 & 121,30 & 135,26 & 144,16 & 150,83 & 156,21 & 174,18 & 194,21 \\
\hline 30 & 98,69 & 109,97 & 117,16 & 122,54 & 126,88 & 141,36 & 157,50 \\
\hline 45 & 77,54 & 86,32 & 91,91 & 96,10 & 99,47 & 110,73 & 123,25 \\
\hline 60 & 64,10 & 71,32 & 75,90 & 79,34 & 82,11 & 91,33 & 101,59 \\
\hline 120 & 38,47 & 42,72 & 45,42 & 47,44 & 49,06 & 54,48 & 60,48 \\
\hline 180 & 27,82 & 30,86 & 32,78 & 34,22 & 35,38 & 39,24 & 43,51 \\
\hline 240 & 21,93 & 24,30 & 25,80 & 26,92 & 27,83 & 30,83 & 34,16 \\
\hline 300 & 18,16 & 20,11 & 21,35 & 22,27 & 23,02 & 25,48 & 28,21 \\
\hline 360 & 15,55 & 17,20 & 18,26 & 19,04 & 19,67 & 21,77 & 24,08 \\
\hline 420 & 13,61 & 15,06 & 15,97 & 16,66 & 17,21 & 19,03 & 21,04 \\
\hline 480 & 12,12 & 13,41 & 14,22 & 14,82 & 15,31 & 16,93 & 18,71 \\
\hline 600 & 9,98 & 11,03 & 11,69 & 12,18 & 12,58 & 13,90 & 15,35 \\
\hline 720 & 8,50 & 9,39 & 9,95 & 10,37 & 10,70 & 11,82 & 13,05 \\
\hline 960 & 6,60 & 7,28 & 7,71 & 8,03 & 8,28 & 9,14 & 10,08 \\
\hline 1200 & 5,41 & 5,96 & 6,31 & 6,57 & 6,78 & 7,48 & 8,24 \\
\hline 1440 & 4,60 & 5,07 & 5,36 & 5,58 & 5,76 & 6,34 & 6,99 \\
\hline
\end{tabular}

Fonte: Zuffo (2009)

Zuffo (2009) destaca a comparação entre as equações de São Paulo e Guarulhos, por Martinez e Magni (1999) e Zuffo (2009), respectivamente, na qual as estimativas das alturas precipitadas são maiores para Guarulhos, o que representa coerência pela proximidade do posto de Cumbica com a Serra da Cantareira, na qual a região está sujeita ao efeito orográfico da mesma, aumentando o volume de precipitações. A Tabela 3 apresenta as diferenças citadas.

Tabela 3 - Diferenças relativas entre os valores das intensidades originais utilizadas para a determinação da Equação de chuvas de São Paulo de Martinez e Magni (1999) e os valores resultantes da Equação de chuva de Guarulhos por Zuffo (2009).

\begin{tabular}{|c|c|c|c|c|c|c|c|}
\hline \multirow{2}{*}{$\begin{array}{l}\text { Duração } \\
\text { (minutos) }\end{array}$} & \multicolumn{7}{|c|}{ TR (anos) } \\
\hline & 5 & 10 & 15 & 20 & 25 & 50 & 100 \\
\hline 10 & $20,0 \%$ & $17,3 \%$ & $16,6 \%$ & $16,4 \%$ & $16,4 \%$ & $17,0 \%$ & $18,5 \%$ \\
\hline 15 & $19,7 \%$ & $16,9 \%$ & $16,2 \%$ & $15,9 \%$ & $15,9 \%$ & $16,5 \%$ & $17,9 \%$ \\
\hline 20 & $19,6 \%$ & $16,7 \%$ & $15,8 \%$ & $15,6 \%$ & $15,5 \%$ & $16,1 \%$ & $17,4 \%$ \\
\hline 30 & $19,4 \%$ & $16,3 \%$ & $15,4 \%$ & $15,1 \%$ & $15,0 \%$ & $15,4 \%$ & $16,7 \%$ \\
\hline 45 & $19,3 \%$ & $16,0 \%$ & $15,0 \%$ & $14,6 \%$ & $14,5 \%$ & $14,8 \%$ & $15,9 \%$ \\
\hline 60 & $19,2 \%$ & $15,8 \%$ & $14,7 \%$ & $14,3 \%$ & $14,1 \%$ & $14,3 \%$ & $15,4 \%$ \\
\hline 120 & $19,3 \%$ & $15,4 \%$ & $14,2 \%$ & $13,6 \%$ & $13,3 \%$ & $13,2 \%$ & $14,0 \%$ \\
\hline 180 & $19,4 \%$ & $15,3 \%$ & $13,9 \%$ & $13,2 \%$ & $12,9 \%$ & $12,6 \%$ & $13,2 \%$ \\
\hline 240 & $19,5 \%$ & $15,2 \%$ & $13,7 \%$ & $13,0 \%$ & $12,6 \%$ & $12,2 \%$ & $12,7 \%$ \\
\hline 300 & $19,6 \%$ & $15,2 \%$ & $13,6 \%$ & $12,8 \%$ & $12,4 \%$ & $11,8 \%$ & $12,3 \%$ \\
\hline 360 & $19,7 \%$ & $15,2 \%$ & $13,5 \%$ & $12,7 \%$ & $12,2 \%$ & $11,6 \%$ & $11,9 \%$ \\
\hline 420 & $19,8 \%$ & $15,2 \%$ & $13,5 \%$ & $12,6 \%$ & $12,1 \%$ & $11,4 \%$ & $11,6 \%$ \\
\hline 480 & $19,9 \%$ & $15,1 \%$ & $13,4 \%$ & $12,5 \%$ & $12,0 \%$ & $11,2 \%$ & $11,4 \%$ \\
\hline 600 & $20,0 \%$ & $15,1 \%$ & $13,3 \%$ & $12,3 \%$ & $11,8 \%$ & $10,9 \%$ & $11,0 \%$ \\
\hline 720 & $20,1 \%$ & $15,1 \%$ & $13,2 \%$ & $12,2 \%$ & $11,6 \%$ & $10,6 \%$ & $10,6 \%$ \\
\hline 960 & $20,3 \%$ & $15,1 \%$ & $13,1 \%$ & $12,0 \%$ & $11,4 \%$ & $10,2 \%$ & $10,1 \%$ \\
\hline 1200 & $20,4 \%$ & $15,1 \%$ & $13,0 \%$ & $11,9 \%$ & $11,2 \%$ & $9,9 \%$ & $9,7 \%$ \\
\hline 1440 & $20,6 \%$ & $15,1 \%$ & $12,9 \%$ & $11,8 \%$ & $11,0 \%$ & $9,6 \%$ & $9,3 \%$ \\
\hline
\end{tabular}

Fonte: Zuffo (2009) 
Conforme Zuffo (2009), as equações para São Paulo não acompanham os mesmos padrões seguidos pelos totais anuais dos três postos, então acredita-se que a equação esteja subestimando as precipitações para a cidade de São Paulo.

Para Zuffo (2015), existem vários ciclos solares que interferem no clima, e um deles, o ciclo de Gleissberg, seria responsável pelo efeito climático conhecido como Efeito José, cujo período médio varia entre de 70 a 100 anos. Esse Efeito José seria, provavelmente, responsável pelo aumento e pela diminuição das precipitações de décadas, tendo sido identificado o período seco de 1936 a
1975 e o úmido de 1976 a 2008 2010. A Fig. 2 apresenta um gráfico do Posto do IAG-USP demonstrando o efeito do aumento da precipitação total anual em $15 \%$, observado entre as médias do primeiro e segundo intervalos anteriormente. Dessa forma, esses dados pluviométricos máximos diários também poderiam sofrer a influência desses períodos. Recomendou-se que se separassem esses períodos para estudos hidrológicos. Na Equação de Martinez e Magni (1999), esses períodos estão misturados, e na Equação de Zuffo (2009) utilizaram-se somente os valores do período úmido do Efeito José.

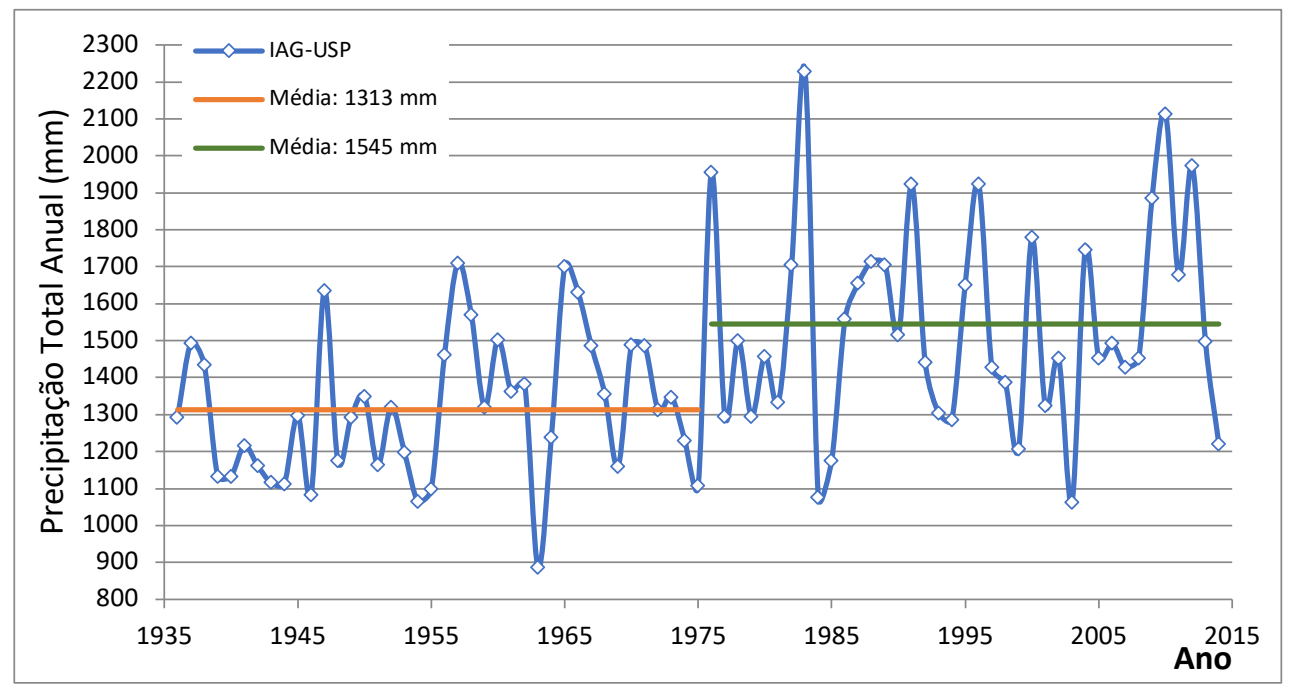

Figura 2 - Série histórica de precipitações do Posto Pluviométrico instalado no IAG - USP, no município de São Paulo, no Parque do Estado. Fonte (Zuffo e Zuffo, 2016).

\section{OBJETIVO}

O objetivo do estudo é comparar os resultados das máximas vazões de duração 120 min e TR 10 e 25 anos, encontradas com a utilização de simulação hidrológica para a bacia do córrego Tiquatira, a partir de valores de precipitação de São Paulo obtidos com as equações de Martinez e Magni (1999) e de Guarulhos, por Zuffo (2009).

\section{METODOLOGIA}

Com a utilização do software Arcmap foi possível utilizar o material fornecido pela EMPLASA, os arquivos de Modelo Digital de Superfície (MDS) e, então, obtiveram-se as declividades ao longo dos talvegues principais, os córregos da Ponte Rasa e Franquinho, afluentes do córrego Tiquatira. 0 cadastro topográfico da região também foi fornecido pela Prefeitura Municipal de São Paulo (PMSP) , com conferência in loco para maior conhecimento das margens do cór- 
rego. $O$ tempo de concentração da bacia foi calculado pelo modelo Cinemático, para cada uma das 3 sub-bacias. Conforme Canholi (2014), os métodos para o cálculo do tempo de concentração estimado recomendam a soma de três parcelas, conforme a Eq. 5.

$t c=t o f+t s c+t c f$

Em que:

tc - tempo de concentração da sub bacia (min); tof - tempo do escoamento superficial laminar "overland flow" (min);

tsc - tempo do escoamento em canais rasos "shallow concentrated flow" (min) e tcf-tempo do escoamento em canais naturais e artificiais "channel flow" (min).

Os resultados são apresentados na Tabela 2

Tabela 2 - tempo de concentração das sub-bacias

\begin{tabular}{|c|c|c|c|}
\hline Sub-bacia & tc (minutos) & L (m) & Área $\mathbf{( k m}^{2}$ ) \\
\hline Franquinho & 88,17 & 6599 & 8,04 \\
\hline Ponte Rasa & 99,77 & 6781 & 7,45 \\
\hline Tiquatira & 10,20 & 3710 & 3,77 \\
\hline
\end{tabular}

A etapa seguinte foi o estudo de uso e ocupação do solo, também com a utilização do Arcmap, que possibilitou a análise por imagem de satélite e, então, identificou-se o total de área urbanizada da bacia. A Fig. 3 ilustra a bacia do córrego Tiquatira e sua área urbanizada.

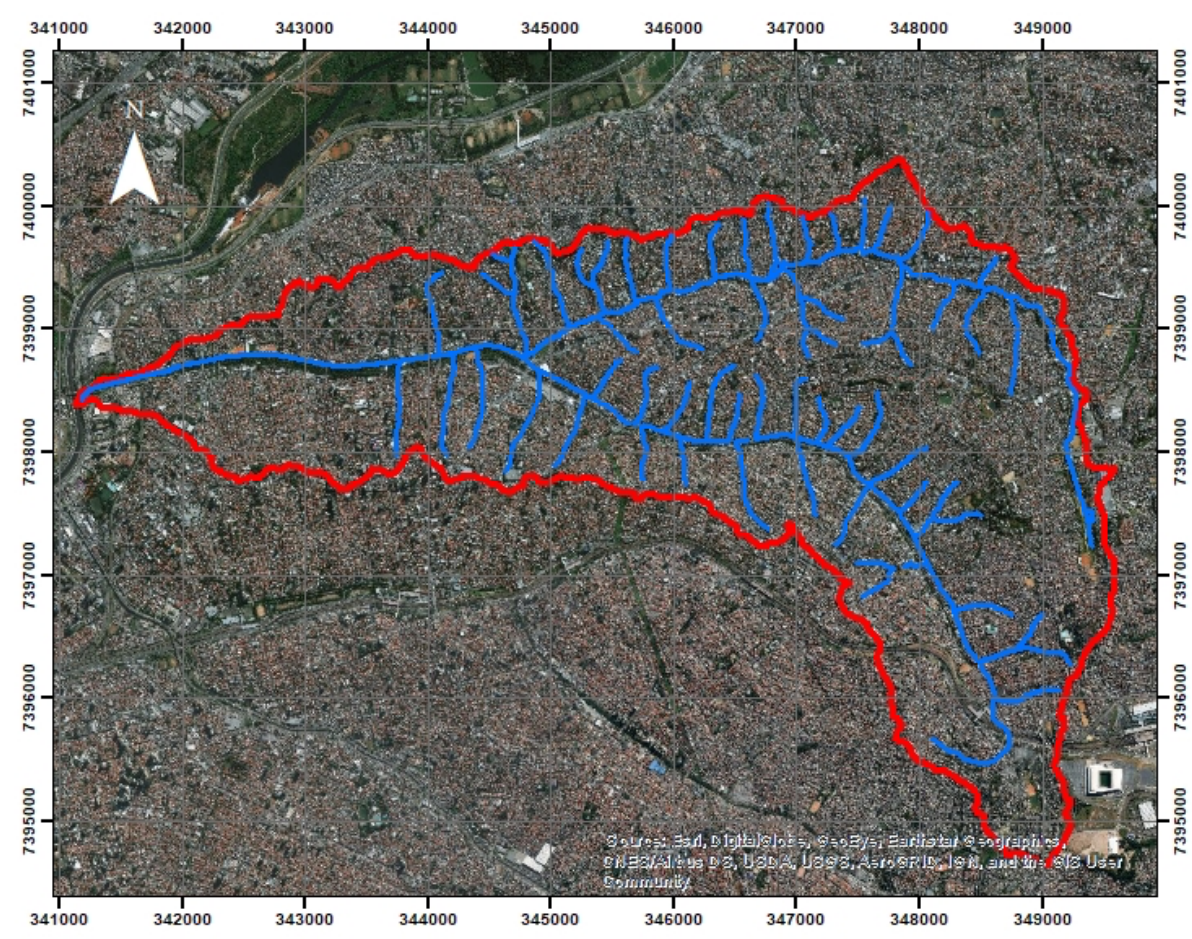

Figura 3 - Imagem de satélite com a localização da bacia do córrego Tiquatira

O método Precipitação-vazão adotado foi o Soil Conservation Service (SCS) desenvolvido pelo Departamento de Agricultura dos Estados Unidos. A fórmula desenvolvida pelo SCS está apresentada na Eq. 6:
$Q=\frac{\left(P_{a c}-0,2 S\right)^{2}}{\left(P_{a c}+0,8 S\right)}$, para $\mathrm{Pac}>0,2 \mathrm{~S}$

Na qual: $Q$ = escoamento superficial direto em $\mathrm{mm} ; \mathrm{P}=$ precipitação em $\mathrm{mm} ; \mathrm{S}=$ retenção potencial do solo em $\mathrm{mm}$. 
O valor de $S$ depende do tipo e da ocupação de solo, e é utilizado para calcular o parâmetro $\mathrm{CN}$ (número da curva), no qual varia entre 0 e 100. Como a bacia do córrego Tiquatira encontra-se em área urbanizada, foi utilizada a Eq. 7.

$C N_{w}=C N_{p e r m}(1-f)+98 f$

Em que:

$\mathrm{CN}_{\mathrm{w}}$ = número da curva composto;

$\mathrm{CN}_{\text {perm }}=$ número da curva da área permeável conforme o tipo de solo do SCS;

$f=$ fração da área impermeável.
Como resultado para a bacia em estudo, considerados $\mathrm{CN}_{\text {perm }} 79$ e f, $86 \%$, o CN determinado foi 95 .

A etapa que envolve a comparação das equações de chuva de São Paulo, de Martinez e Magni (1999) e Guarulhos, por Zuffo (2009) é a do desenvolvimento dos Hietogramas, realizados para os períodos de retorno (TR) de 10 e 25 anos. A duração de chuva de 2 horas foi adotada a partir de uma aproximação do tempo de concentração calculado anteriormente. No presente trabalho, os hietogramas foram desenvolvidos pelo método de Huff para bacias urbanas, considerando $1^{\circ}$ Quartil. A Tabela 3 apresenta a distribuição média durante um evento de chuva.

Tabela 3 - Distribuição Média do Tempo de Chuva em áreas de 26 a 130 quilômetros quadrados.

\begin{tabular}{|c|c|c|c|c|}
\hline \% CUMULATIVA & $1^{\circ}$ QUARTIL & $2^{\circ}$ QUARTIL & $3^{\circ}$ QUARTIL & $4^{\circ}$ QUARTIL \\
\hline $5 \%$ & $12 \%$ & $3 \%$ & $2 \%$ & $2 \%$ \\
\hline $10 \%$ & $25 \%$ & $6 \%$ & $5 \%$ & $4 \%$ \\
\hline $15 \%$ & $38 \%$ & $10 \%$ & $8 \%$ & $7 \%$ \\
\hline $20 \%$ & $51 \%$ & $14 \%$ & $12 \%$ & $9 \%$ \\
\hline $25 \%$ & $62 \%$ & $21 \%$ & $14 \%$ & $11 \%$ \\
\hline $30 \%$ & $69 \%$ & $30 \%$ & $17 \%$ & $13 \%$ \\
\hline $35 \%$ & $74 \%$ & $40 \%$ & $20 \%$ & $15 \%$ \\
\hline $40 \%$ & $78 \%$ & $52 \%$ & $23 \%$ & $18 \%$ \\
\hline $45 \%$ & $81 \%$ & $63 \%$ & $27 \%$ & $21 \%$ \\
\hline $50 \%$ & $84 \%$ & $72 \%$ & $33 \%$ & $24 \%$ \\
\hline $55 \%$ & $86 \%$ & $78 \%$ & $42 \%$ & $27 \%$ \\
\hline $60 \%$ & $88 \%$ & $83 \%$ & $55 \%$ & $30 \%$ \\
\hline $65 \%$ & $90 \%$ & $87 \%$ & $69 \%$ & $34 \%$ \\
\hline $70 \%$ & $92 \%$ & $90 \%$ & $79 \%$ & $40 \%$ \\
\hline $75 \%$ & $94 \%$ & $92 \%$ & $86 \%$ & $47 \%$ \\
\hline $80 \%$ & $95 \%$ & $94 \%$ & $91 \%$ & $57 \%$ \\
\hline $85 \%$ & $96 \%$ & $96 \%$ & $94 \%$ & $74 \%$ \\
\hline $90 \%$ & $97 \%$ & $97 \%$ & $96 \%$ & $88 \%$ \\
\hline $95 \%$ & $98 \%$ & $98 \%$ & $98 \%$ & $95 \%$ \\
\hline
\end{tabular}

O fluxograma a seguir ilustra as etapas realizadas para a obtenção dos resultados

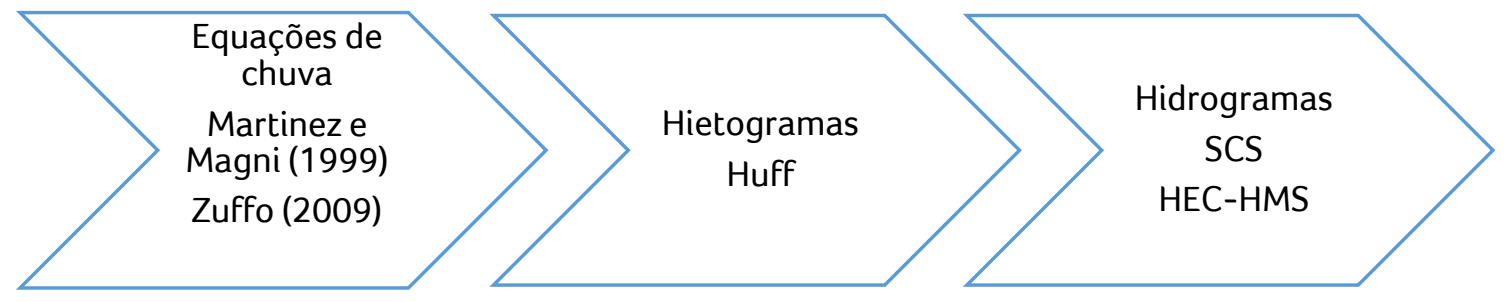




\section{RESULTADOS}

As tabelas referentes aos hietogramas, para uma duração de chuva de 120 minutos e períodos de retorno de 10 e 25 anos, são apresentadas a seguir pelas Tabelas 4, 5, 6 e 7.

Tabela 4 - Hietograma para a Equação de Martinez e Magni (1999) (TR 10 anos).

\begin{tabular}{|c|c|c|}
\hline Duração (min) & Altura Acumulada (mm) & $\begin{array}{c}\text { Altura } \\
\text { desacumulada (mm) }\end{array}$ \\
\hline 6 & 8,671 & 8,671 \\
\hline 12 & 18,065 & 9,394 \\
\hline 18 & 27,459 & 9,394 \\
\hline 24 & 36,852 & 9,394 \\
\hline 30 & 44,801 & 7,949 \\
\hline 36 & 49,859 & 5,058 \\
\hline 42 & 53,472 & 3,613 \\
\hline 48 & 56,362 & 2,890 \\
\hline 54 & 58,530 & 2,168 \\
\hline 60 & 60,698 & 2,168 \\
\hline 66 & 62,143 & 1,445 \\
\hline 72 & 63,588 & 1,445 \\
\hline 78 & 65,034 & 1,445 \\
\hline 84 & 66,479 & 1,445 \\
\hline 90 & 67,924 & 1,445 \\
\hline 96 & 68,646 & 0,723 \\
\hline 102 & 69,369 & 0,723 \\
\hline 108 & 70,092 & 0,723 \\
\hline 114 & 70,814 & 0,723 \\
\hline 120 & 72,259 & 1,445 \\
\hline & & 72,259 \\
\hline & & \\
\hline
\end{tabular}

Tabela 5 - Hietograma para a Equação de Martinez e Magni (1999) (TR 25 anos).

\begin{tabular}{|c|c|c|}
\hline Duração (min) & Altura Acumulada (mm) & $\begin{array}{c}\text { Altura } \\
\text { desacumulada }(\mathrm{mm})\end{array}$ \\
\hline 6 & 10,208 & 10,208 \\
\hline 12 & 21,268 & 11,059 \\
\hline 18 & 32,327 & 11,059 \\
\hline 24 & 43,386 & 11,059 \\
\hline 30 & 52,744 & 9,358 \\
\hline 36 & 58,699 & 5,955 \\
\hline 42 & 62,952 & 4,254 \\
\hline 48 & 66,355 & 3,403 \\
\hline 54 & 68,907 & 2,552 \\
\hline 60 & 71,459 & 2,552 \\
\hline 66 & 73,161 & 1,701 \\
\hline 72 & 74,862 & 1,701 \\
\hline 78 & 76,564 & 1,701 \\
\hline 84 & 78,265 & 1,701 \\
\hline 90 & 79,966 & 1,701 \\
\hline 96 & 80,817 & 0,851 \\
\hline 102 & 81,668 & 0,851 \\
\hline 108 & 82,518 & 0,851 \\
\hline 114 & 83,369 & 0,851 \\
\hline 120 & 85,071 & 1,701 \\
\hline \multicolumn{2}{|r|}{ Total } & 85,071 \\
\hline
\end{tabular}

Tabela 6 - Hietograma para a Equação de Zuffo (2009) (TR 10 anos).

\begin{tabular}{|c|c|c|}
\hline Duração (min) & Altura Acumulada (mm) & $\begin{array}{c}\text { Altura } \\
\text { desacumulada }(\mathrm{mm})\end{array}$ \\
\hline 6 & 10,254 & 10,254 \\
\hline 12 & 21,362 & 11,108 \\
\hline 18 & 32,470 & 11,108 \\
\hline 24 & 43,578 & 11,108 \\
\hline 30 & 52,978 & 9,399 \\
\hline 36 & 58,959 & 5,981 \\
\hline 42 & 63,231 & 4,272 \\
\hline 48 & 66,649 & 3,418 \\
\hline 54 & 69,213 & 2,563 \\
\hline 60 & 71,776 & 2,563 \\
\hline 66 & 73,485 & 1,709 \\
\hline 72 & 75,194 & 1,709 \\
\hline 78 & 76,903 & 1,709 \\
\hline 84 & 78,612 & 1,709 \\
\hline 90 & 80,321 & 1,709 \\
\hline 96 & 81,175 & 0,854 \\
\hline 102 & 82,030 & 0,854 \\
\hline 108 & 82,884 & 0,854 \\
\hline 114 & 83,739 & 0,854 \\
\hline 120 & 85,448 & 1,709 \\
\hline \multicolumn{2}{|r|}{ TOTAL } & 85,448 \\
\hline
\end{tabular}

Tabela 7 - Hietograma para a Equação de Zuffo (2009) (TR 25 anos).

\begin{tabular}{|c|c|c|}
\hline Duração (min) & Altura Acumulada (mm) & $\begin{array}{c}\text { Altura } \\
\text { desacumulada (mm) }\end{array}$ \\
\hline 6 & 11,776 & 11,776 \\
\hline 12 & 24,532 & 12,757 \\
\hline 18 & 37,289 & 12,757 \\
\hline 24 & 50,046 & 12,757 \\
\hline 30 & 60,840 & 10,794 \\
\hline 36 & 67,709 & 6,869 \\
\hline 42 & 72,616 & 4,906 \\
\hline 48 & 76,541 & 3,925 \\
\hline 54 & 79,485 & 2,944 \\
\hline 60 & 82,429 & 2,944 \\
\hline 66 & 84,391 & 1,963 \\
\hline 72 & 86,354 & 1,963 \\
\hline 78 & 88,317 & 1,963 \\
\hline 84 & 90,279 & 1,963 \\
\hline 90 & 92,242 & 1,963 \\
\hline 96 & 93,223 & 0,981 \\
\hline 102 & 94,204 & 0,981 \\
\hline 108 & 95,186 & 0,981 \\
\hline 114 & 96,167 & 0,981 \\
\hline 120 & 98,130 & 1,963 \\
\hline & & 98,130 \\
\hline & & \\
\hline & & \\
\hline
\end{tabular}

Comparando os resultados da altura acumulada para duração de $120 \mathrm{~mm}$, pode-se observar, para os períodos de retorno de 10 e 25 anos, que a Equação de Zuffo (2009) para Guarulhos totali- 
zou $18 \%$ e $15 \%$, respectivamente, a mais no total da altura de chuva em relação à Equação de Martinez e Magni (1999), coincidindo com o mesmo percentual de aumento das precipitações totais anuais, no posto pluviométrico do IAG-USP, conforme mostrado anteriormente na Fig. 2.

Aplicado o método Soil Conservation Service (SCS) com a utilização dos respectivos hietogramas obtidos conforme Itens 2 e 3 no software HEC-HMS, obtiveram-se os seguintes resultados de vazão de pico para a bacia do córrego Tiquatira, apresentados na Tabela 8.
Tabela 8 - Comparativo entre as vazões de pico.

\begin{tabular}{|c|c|c|}
\hline Equação & TR (anos) & Vazão $\left(\mathbf{m}^{\mathbf{3}} \mathbf{s}\right)$ \\
\hline $\begin{array}{c}\text { Martinez e Magni } \\
\text { (1999) }\end{array}$ & 10 & 119,4 \\
\hline São Paulo & 25 & 142,6 \\
\hline $\begin{array}{c}\text { Zuffo } \\
\text { (2009) } \\
\text { Guarulhos }\end{array}$ & 10 & 143,3 \\
\cline { 2 - 3 } & 25 & 166,3 \\
\hline
\end{tabular}

Verifica-se nos Gráficos 1 e 2 o comportamento dos hidrogramas gerados pelo software HEC-HMS, para os períodos de recorrência de 10 e 25 anos, respectivamente.

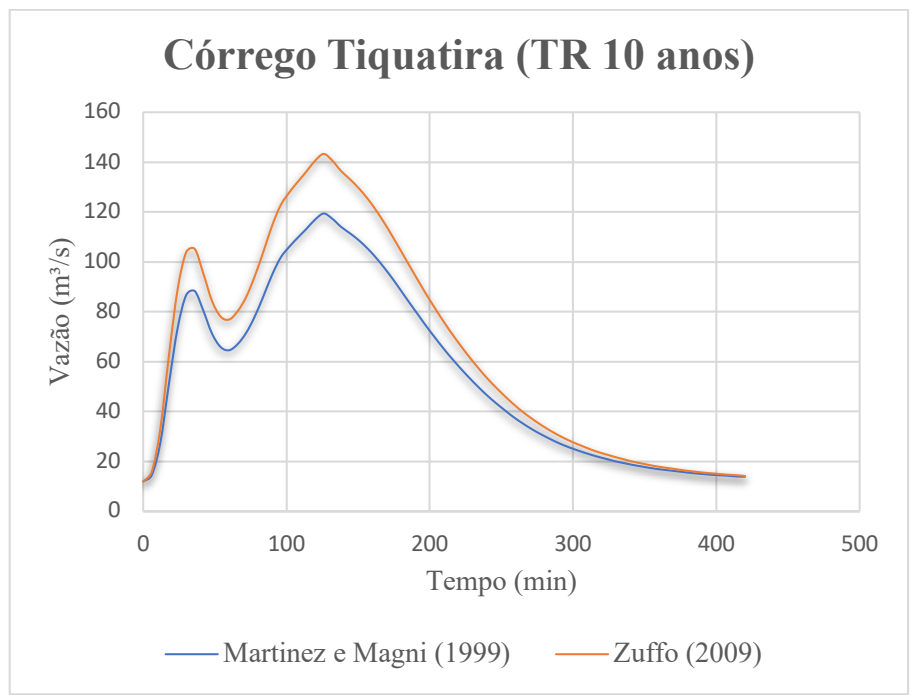

Gráfico 1 - Hidrogramas comparativos para TR 10 anos.

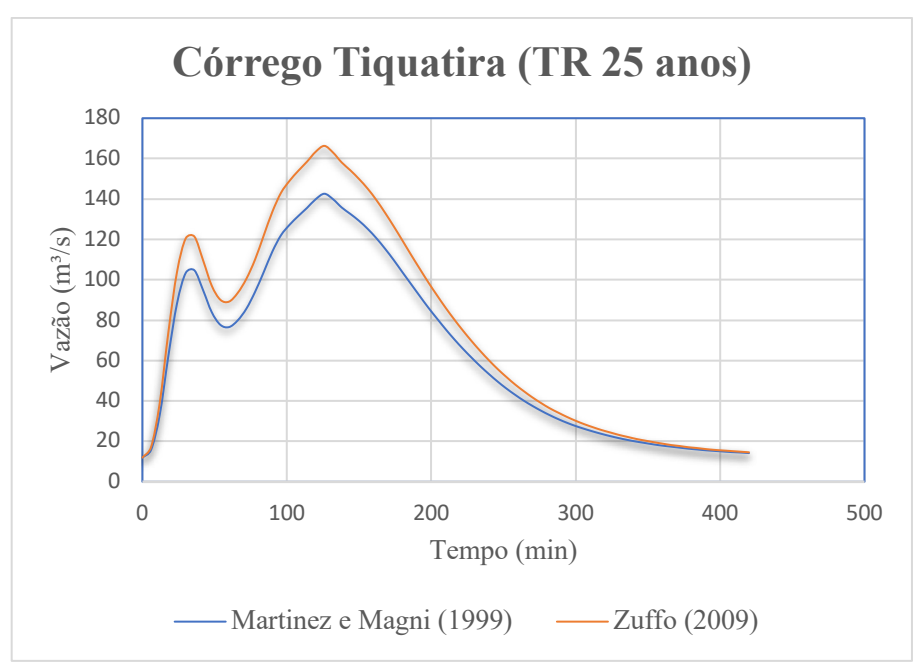

Gráfico 2 - Hidrogramas comparativos para TR 25 anos. 


\section{CONCLUSÃO}

Ao analisar os resultados, pode-se concluir que, para o período de retorno de 10 anos, a vazão de pico utilizando a equação para a cidade de Guarulhos, por Zuffo (2009), resultou em um valor de $20 \%$ maior do que a vazão resultante calculada com a equação de São Paulo, por Martinez e Magni (1999). Nota-se também para TR 25 anos que a vazão de pico calculada com a equação de Zuffo (2009) foi $17 \%$ superior do que utilizando a equação de Martinez e Magni (1999). Pode-se afirmar que, no caso da região que contempla a bacia do córrego Tiquatira, ao utilizar a equação de Martinez e Magni (1999), mesmo que o posto pluviográfico de referência no qual a equação foi desenvolvida esteja na mesma cidade, as estruturas hidráulicas podem ser subestimadas e não comportar o volume de chuva da região, invalidando a solução proposta com a continuidade das inundações.

Torna-se, então, válido o comparativo entre diferentes equações de chuvas, desde que recomendadas pelo DAEE, a fim de não subestimar os projetos de drenagem urbana, com o objetivo de estar em favor da segurança. Mais estudos devem ser dedicados ao Efeito José e seus feitos nas precipitações máximas diárias e totais anuais, assim como sua influência nas vazões dos rios.

\section{CONTRIBUIÇÃO DOS AUTORES}

Todos os autores contribuíram de forma igualitária.

\section{REFERÊNCIAS}

CANHOLI, A. P. Drenagem urbana e controle de enchentes. 2. ed. São Paulo: Oficina de Textos, 2014. 384 p. ISBN: 978-85-7975-160-8 CHEN, C. L. Rainfall Intensity - duration - frequency formulas. Journal of Hydraulic Engineering - ASCE, Vol. 109, n. 12, p. $1603-$ 21, 1983.

CHOW, V. T. Section 8-I. Statistical and probability analysis of hydrologic data. Part I: Frequency Analysis. In: Handbook of Applied Hydrology. Mcgraw Hill. USA: pp. 2-37, 1954.

HUFF, F. Time Distributions of Heavy Rainstorms in Illinois Time Distributions of Heavy Rainstorms in Illinois. Water Survey Campaign, p. 22, 1990.

NUNES, D. M.; SILVA, L. P. DA; FONSECA, P. L. DA. Avaliação do papel dos telhados verdes no desenho e desenvolvimento urbano de baixo impacto ambiental e no controle de enchentes na Cidade do Rio de Janeiro. Labor e Engenho, v. 11, n. 3, p. 374, 2017. https:// doi.org/10.20396/labore.v11i3.8648820

SÃO PAULO. Departamento de Águas e Energia Elétrica; Centro Tecnológico de Hidráulica. Secretaria de Saneamento e Recursos Hídricos (Org.). Precipitações Intensas na Bacia do Alto Tietê. 2018.

TUCCI, C. E. M.; PORTO, R. L. L.; MÁRIO T. DE BARROS. Drenagem Urbana. 1a ed. Porto Alegre: [s.n.]. ISBN: 978-85-7025-364-4

ZUFFO, A. C. Determinação da equação de chuvas de Guarulhos, São Paulo. Relatório Técnico - LADSEA - FEC - UNICAMP. 2009.

ZUFFO, A. C. O Sol: O motor das variabilidades climáticas. Revista DAE, , S.L.], v. 63, n. 198, p. 6-24, 2015. Revista DAE. http://dx.doi. org/10.4322/dae.2014.142

ZUFFO, A. C. e ZUFFO, M. S. R. Gerenciamento de Recursos Hídricos: Conceituação e Constextualização. ELSEVIER. ISBN: 978-85-352-7703-6 